\title{
Intelligent Paging Strategies for Third Generation Mobile Telecommunication Systems
}

George L. Lyberopoulos, Member, IEEE, John G. Markoulidakis, Dimitrios V. Polymeros, Dimitrios F. Tsirkas, and Efstathios D. Sykas, Member, IEEE

\begin{abstract}
Location management procedures, in conjunction with the millions of users expected to subscribe to third generation mobile telecommunication systems, will generate a huge signaling load. In this paper, we propose a method which aims at the reduction of signaling overhead on the radio link produced by the paging procedure. The key idea is the application of a multiple step paging strategy which operates as follows: At the instance of a call terminating to a mobile user who roams within a certain location area, paging is initially performed in a portion of the location area (the paging area) that the so-called "paging related information" indicates. On no paging response, the mobile user is paged in the complementary portion of the location area-this phase can be completed in more than one (paging) step. Various "paging related information" elements (e.g., recent interaction information, high mobility flag, etc.) can be used and several "intelligent" paging strategies can be defined. Representative paging strategies are analyzed in terms of network performance and quality of service (paging signaling, paging delay, processing power requirements) via a simulation tool which models a realistic city area environment.
\end{abstract}

\section{INTRODUCTION}

QECOND generation cellular mobile telecommunication

Q systems offering international roaming in several European countries are realized by the Global System for Mobile Communications (GSM). It is envisaged that GSM will run out of capacity before the end of the century. At that time, third generation mobile telecommunication systems will come into foreground. Among them are: the Universal Personal Telecommunications (UPT) and the Universal Mobile Telecommunication System (UMTS). UPT enables access to telecommunication services while allowing Personal Mobility. "It enables each UPT user to participate in a user-defined set of subscribed services and to initiate and receive calls on the basis of a unique, personal, network independent UPT Number across multiple networks at any terminal, fixed, [movable] or mobile irrespective of the geographic location, limited only by terminal and network capabilities and restrictions imposed by the network provider" [1]. UMTS will offer a plethora of mobile telecommunication services (e.g., voice, low and high bitrate data and video) to Mobile Users (MU) via a range of Mobile Terminals (MT) operating in both public and private

Manuscript received July 27, 1993; revised March 28, 1994. This paper has been partially funded by EU through the RACE 2066 MObile NETwork (MONET) project. The paper does not present the views of the project as a whole, but those of the authors.

The authors are with the Department of Electrical and Computer Engineering, National Technical University of Athens, 15773, Athens, Greece. IEEE Log Number 9411292. environments. ${ }^{1}$ The ultimate aim is that mobile users should be able to initiate and receive calls with "anyone," "anywhere," "anytime" [2]. UMTS can be conceived as a mixture of public and private networks combined in such a way so as to offer mobility and continuity of ongoing calls. Contrary to what happens in the second generation mobile systems, in the third generation systems mobility functionality is integrated into the fixed network (possibly the B-ISDN).

\section{A. Tracking Mobile Users}

In second generation mobile communication systems, the issue of location finding is treated as follows [3]. The system coverage area is divided into geographical areas, called Location Areas (LA) and the network keeps track the location of every "switched-on" MT with the accuracy of an LA. User "location information" (i.e., the identity of the LA within which the user currently roams) is stored in the network database. In order to maintain the consistency of the "location information," an update transaction is triggered whenever a "switched-on" MT crosses LA boundaries (location updating procedure). ${ }^{2}$ At the instance of a call, a locate database transaction is triggered so as to retrieve the user "location information." Then, the MT is paged inside the whole LA simultaneously; that is, the Paging Area (PA) is equal to the LA. $^{3}$

In third generation mobile telecommunication systems the same approach seems to be followed [2]. However, LA planning (size, shape, and configuration of LA's) is of greater importance, especially, in densely populated areas e.g., large cities, where both the density and the mobility of users is high. In such environments, the trade-off between the location updating and paging related signaling load is the main performance optimization problem during LA planning. To assess this trade-off we consider the following two examples: a) the division of a city area into a low number of large LA's results in a moderate number of location updates and a huge amount of paging signaling per LA, while b) the division of a city area into a large number of small-sized LA's

\footnotetext{
${ }^{1}$ The terminals can range from basic low cost simple types, through handheld or vehicle mounted devices up to portable B-ISDN terminals.

${ }^{2}$ The location updating procedure involves a database transaction to update the user "location information." The exchange of the signaling information is performed via a single Base Station.

${ }^{3}$ Paging messages are broadcast via all base stations of the LA and possibly more than once. The outcome of the paging procedure is the establishment of a wireless signaling association between the MT and the network (i.e., the nearest base station).
} 
results in a huge number of location updates and a moderate amount of paging signaling per LA. Algorithms for optimal LA planning are proposed in [4]. Other studies propose the use of multi-layer LA's [5], [6] in order to effectively reduce the location updating cost. In [7] the concept of reporting centers-instead of the conventional LA's-is introduced. The selection of the appropriate set of reporting centers is based on the minimization of both the location updating and paging costs.

\section{B. The Concept of "Intelligent" Paging}

In this paper, we propose a strategy ("intelligent" paging) which aims at the significant reduction of the paging signaling load on the radio link. The "intelligent" paging strategy is shortly described as follows:

At the instance of a call terminating to an MU which roams within a certain LA, paging is initially performed within a portion of the LA (this LA portion constitutes the PA). The selection of a "proper" PA is based on the so-called "paging related information." On no paging response, the MU is paged in the complementary portion of the LA-this phase can be completed in more than one (paging) step.

The effectiveness of the "intelligent" paging strategy-as far as the paging signaling load is concerned-is directly related to the capability of the network to accurately predict the called MU location. From the performance point of view, the following requirements should be fulfilled: ${ }^{4}$

- The quality of service in terms of paging delay should be similar to the "GSM-like" approach for the majority of calls.

- The extra processing required to exploit the "paging related information" should not be considerable.

Although the proposed method requires some additional storage (see "paging related information"), it does not impose any additional transaction to the network database since the retrieval of the "paging related information" can be performed in conjunction with the retrieval of the user profile during the call set-up phase. Current studies [8], [9] indicate that the database implementation cost depends on the number of locate and update transactions. As proposed in [10], information related to the user mobility behavior can be also used so as to improve the database performance. It is important to note that "intelligent" paging operates under any database implementation scheme [8]-[10] (either utilizing user mobility related information or not). This is because paging is always performed after the completion of the locate transaction. An additional advantage of the proposed paging method is that the resulting reduction of the paging cost, can further improve the results of LA planning techniques proposed in [4]-[6] in the sense that "intelligent" paging allows for the definition of larger LA's (e.g., one LA may cover a whole city area). This in turn leads to the reduction of the corresponding number of locate transactions. From this point of view, "intelligent" paging can also be proved fruitful for

\footnotetext{
4"Accuracy" can be expressed as the percentage of calls for which paging is completed successfully in the first paging step (typical value $90 \%$ ).
}

the database performance as it allows for the reduction of the number of update transactions.

The material included in this paper is organized as follows. In Section II, representative "intelligent" paging strategies are presented. In Section III, an algorithm for the efficient use of the "paging related information" elements is proposed. In Section IV, various "intelligent" paging scenarios are evaluated in terms of: a) the percentage of successful paging attempts and b) the paging related signaling load they produce versus complexity. Finally, in Section V some concluding remarks are drawn.

\section{II. "INTELLIGENT" PAGING}

"Intelligent" paging is a multiple step paging strategy which utilizes the so-called "paging related information" so as to determine the proper PA within which a called MT currently roams. ${ }^{5}$ A PA is defined as a set of Base Stations for Paging (BSP). ${ }^{6}$ The grouping of BSP's into PA's may be: a) fixed (long-term changes are allowed), b) semi-dynamic (adjacent BSP's can be grouped into PA's while short term changes are allowed) or c) fully-dynamic (any BSP combination is allowed). In this paper we adopt the fully-dynamic BSP grouping approach - it seems to offer the greater flexibility as far as the PA size is concerned-and we concentrate on the derivation of paging scenarios according to the available "paging related information."

The application of "intelligent" paging includes the event of paging failures due to unsuccessful predictions. In such cases, another step is (or multiple steps are) required, i.e., the called MU is paged in another portion of the LA. Continuous unsuccessful paging attempts may lead to unacceptable network performance in terms of paging delay. To minimize the number of paging steps, the network should guarantee that the probability the first paging step is successful is high (typical value e.g., 90\%). We will refer to this probability as the Probability of Successful First paging Step (PSFS).

The PSFS depends heavily on the mobility conditions which characterize the LA in a certain daytime period. For example, rush hour tends to lower the PSFS since the location of the moving MU's continuously changes versus time, being thus ambiguous. On the other hand, accurate predictions are likely during low or ordinary mobility time periods, e.g., working hours, night hours.

It is important to notice that "intelligent" paging techniques do not preclude the option of applying a single paging step, e.g., in the cases of emergency calls, when paging information is obsolete, unavailable or not valid (e.g., due to a network or terminal failure), etc.

\footnotetext{
${ }^{5}$ In contrast to the single step paging strategy ("GSM-like" approach).

${ }^{6}$ The paging messages can be broadcast either by the Standard Base Transceiver Stations (BTS) or by Dedicated Base Stations for Paging (DBSP). A DBSP is a Base Station which exclusively broadcasts paging messages over an area usually larger than a single cell. The location of each DBSP as well as the number of DBSP's per LA depends on propagation and traffic characteristics of the environment. In the following, we will refer to Base Stations for Paging (BSP), to denote either a BTS or a DBSP.
} 


\section{A. Elements of the "Paging Related Information"}

The main criteria used to derive the elements of the "paging related information" are:

- The information elements should describe the major characteristics of the user mobility behavior.

- The information elements should be collected by the network without causing extra signaling or specific terminal operations. The employment of "intelligent" paging should be transparent to the MT.

- The user privacy should be protected.

Based on the above criteria we may identify two "paging related information" components: ${ }^{7}$

1) "MU-related" information that reflects the mobility behavior of each individual MU

- A flag which indicates whether an $\mathrm{MU}$ is a high mobility user (e.g., taxi driver, etc.) or an ordinary/low mobility user (e.g., working person, housekeeper, etc.)

- The identity of the BSP which corresponds to the area where the called MU had the most recent interaction. The time when the MU had the most recent interaction is also stored.

- The identities of the BSP's which cover the socalled movement attraction points of an $\mathrm{MU}^{8}$ and approximately the hours the MU is likely to spend there.

\section{2) "Non-MU-related" information}

- The current mobility conditions, i.e., rush hour, ordinary mobility hour, etc.

- The BSP Topology information.

\section{B. "Intelligent" Paging Scenarios}

Depending on the available "paging related information" elements, various "intelligent" paging scenarios can be envisaged. A representative set is shown in Table I. The following paragraphs describe how the "paging related information" elements are exploited for each paging scenario. Indicative performance results are given in Section IV.

1) Scenario 1: The fundamental assumption here is that the network stores the MT location (the identity of the BSP) at which the MT had the most recent interaction (e.g., the occurrence of a terminating or originating call, a location update, etc.). Then, upon call arrival, the network performs paging within the BSP area which the most recent interaction information indicates. Obviously, high PSFS values can be achieved for ordinary mobility user groups (e.g., working persons, housekeepers) and only for specific periods (e.g.,

\footnotetext{
${ }^{7}$ We do not claim that the above information element list is an exhaustive one.

${ }^{8}$ The movement attraction points represent locations where a specific MU resides for a remarkable time period, e.g., the user residence, the user workplace, etc. In addition, other specific locations that an $\mathrm{MU}$ visits on an everyday basis (e.g., a gym, a library, etc.) could be identified. This information may be provided by the user at subscription time. It is not necessary that the user provides the exact addresses, it suffices to indicate areas. Alternatively, the collection of such information may be based on
} network statistics. This however increases the complexity of the system.
TABLE I

"Intelligent" Paging Scenarios

\begin{tabular}{|l|c|c|c|c|c|}
\cline { 2 - 6 } \multicolumn{1}{c|}{} & $\begin{array}{c}\text { Recent } \\
\text { Interaction }\end{array}$ & $\begin{array}{c}\text { Degree of } \\
\text { Mobility }\end{array}$ & $\begin{array}{c}\text { MU } \\
\text { Attraction } \\
\text { Points }\end{array}$ & $\begin{array}{c}\text { Time Zone } \\
\text { Information }\end{array}$ & $\begin{array}{c}\text { BSP } \\
\text { Topology }\end{array}$ \\
\hline Scenario 1 & $\bullet$ & & & & \\
\hline Scenario 2 & $\bullet$ & $\bullet$ & & & \\
\hline Scenario 3 & $\bullet$ & $\bullet$ & $\bullet$ & & \\
\hline Scenario 4 & $\bullet$ & $\bullet$ & $\bullet$ & $\bullet$ & \\
\hline Scenario 5 & $\bullet$ & $\bullet$ & $\bullet$ & $\bullet$ & $\bullet$ \\
\hline
\end{tabular}

working hours). On the contrary, the recent interaction information alone cannot guarantee high PSFS values for high mobility users (e.g., taxis, transportation vehicles, etc.) due to the continuous user motion irrespective of the day time. The same applies for all user categories during rush hours.

2) Scenario la: This is a variation of Scenario 1. The fundamental assumption here is that the network also stores the time when the most recent interaction occurred. Thus, unsuccessful paging attempts can be avoided by simply comparing the age of the most recent interaction with a certain time threshold ("decision threshold"). Scenario la operates as follows: If the network concludes that the recent interaction information is obsolete (or not valid), a single step paging strategy is applied, otherwise paging is performed within the area which the recent interaction information indicates. To further improve the PSFS, the "decision threshold" may: a) adapt dynamically to the current mobility and traffic conditions, (e.g., rush hour, busy hour) and/or b) vary depending on the degree of user mobility (e.g., high, ordinary mobility users). The issue here is the "optimal" selection of the "decision threshold" for proper operation.

3) Scenario 2: According to this scenario, various MU groups can be identified according to their degree of mobility (i.e., number of movements per day), for example:

- High Mobility MUs (e.g., taxis, transportation vehicles, etc.) and

- Ordinary Mobility MUs (e.g., working persons, housekeepers, etc.)

We assign to each MU group different "decision thresholds" with respect to the recent interaction information. Scenario 2 operates as follows: At call set-up, the mobility group of the called MU is retrieved. If the called MU belongs to the High Mobility group, a single step paging strategy is applied, unless a very recent interaction information is available and usable. If Ordinary Mobility MU, the BSP indicated by the most recent interaction information is selected. Scenario 2 can provide accurate predictions for high mobility users only if we assign to them a very short "decision threshold."

4) Scenario 2a: A variation of Scenario 2 is that recent interaction information for high mobility users is not utilized. That is, whenever a high mobility MU is called the network employs the single step paging strategy.

5) Scenario 3: At call set-up, the "recent interaction," the "degree of mobility" and the "Movement Attraction Points" information elements are retrieved. Then, paging is performed within the BSP's the Movement Attraction Point information 


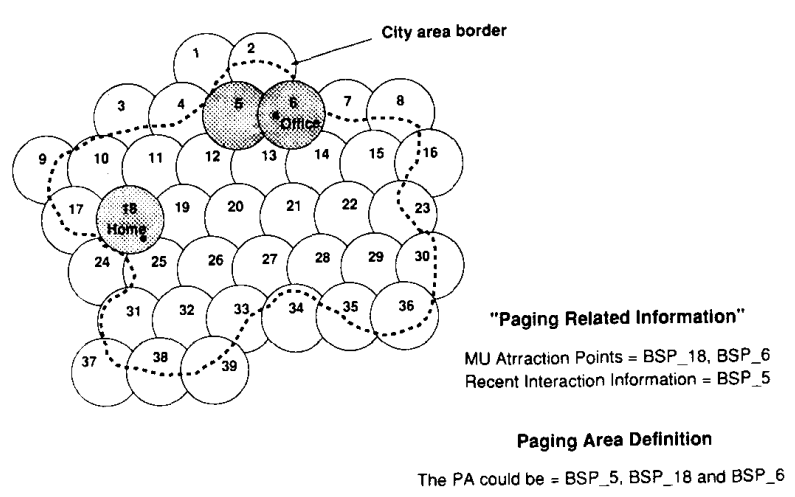

Fig. 1. Selection of the paging area based on the "movement attraction points" and recent interaction information if not obsolete.

indicates. ${ }^{9,10}$ The use of recent interaction information may: a) provide additional helpful information indicating some unusual MU movement and b) confirm that the called MU is located at the "relevant" movement attraction point. An example of how to determine the PA based on the movement attraction points and the recent interaction information for a terminating call to an ordinary mobility MU is shown in Fig. 1.

6) Scenario 4: According to this scenario various mobility time zones can be identified, such as: ${ }^{11}$

- High Mobility Time Zones. During high mobility time zones, the majority of the MU's are in motion (e.g., rush hours).

- Ordinary Mobility Time Zones. During ordinary mobility time zones, most of the MU's are located at some of the movement attraction points (e.g., in the morning hours most people are at their offices, while in the afternoon hours most of them are at home).

- Low Mobility Time Zones. These time zones are characterized by low mobility (e.g., night hours).

The aim of scenario 4 is two-fold: a) to reduce the PA size at certain day time periods during which the user is likely to reside within a certain movement attraction point (e.g., working persons during working hours) and b) to increase the PSFS during high mobility time zones by adopting certain "decision thresholds" for all MU categories.

The characterization of a mobility time zone can be based either on pre-defined timetables or on real measurements (network statistics). ${ }^{12}$ The latter would be preferable since mobility and traffic conditions are not always the expected ones. In this case, the current mobility time zone can be determined by monitoring the PSFS value. A procedure to accomplish this might be the following: whenever the network

\footnotetext{
9 For high mobility MU's (e.g., taxis), Movement Attraction Point information is not considered relevant due to their continuous movement. For such users paging is performed within the whole LA, unless recent interaction information is not obsolete.

${ }^{10}$ Note that change of a Movement Attraction Point will result in continuous unsuccessful paging attempts. Such unwanted situations should be monitored by the network for a proper operation.

${ }^{11}$ A mobility time zone characterizes the current mobility conditions within a certain LA.

${ }^{12}$ Additional types of time zones can be envisaged for special occasions (e.g., weekends, holidays) during which the MU movements are extraordinary.
}

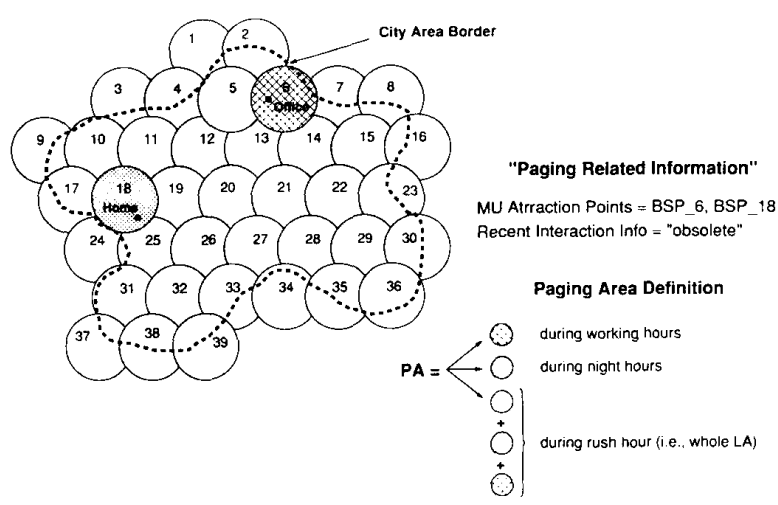

Fig. 2. Selection of the paging area based on "movement attraction points" and "time zones information" for an ordinary mobility MU.

deduces that PSFS probability becomes lower than a certain threshold (which expresses the mobility conditions during the current time zone), it alters the algorithm used for the prediction of the "proper" PA (e.g., increases the PA size).

The mobility time zone information can be utilized by the network as shown in the following example. Assume that an ordinary mobility MU is called. If the current time zone is an ordinary or low mobility time zone, the MU will be first paged within the PA indicated by the "MU-related" paging information (i.e., movement attraction points, most recent interaction information). If there is no paging response, then the MU is paged in the remaining LA (two step paging strategy). As far as high mobility MU's are concerned, when information regarding a very recent interaction is not available, a single paging step strategy is applied. Fig. 2 depicts the PA selection for terminating calls to an ordinary mobility $\mathrm{MU}$ during: a) working hours, b) night hours, and c) during the rush hour. In this example, we assume that the most recent interaction information is obsolete and not used.

7) Scenario 5: The BSP topology information represents some kind of "network-related" paging information. It provides means to improve the PSFS by simply increasing the PA size which the "paging related information" indicates (original PA). This is accomplished by adding adjacent BSP areas to the original PA. The BSP topology information can be utilized whenever the network detects low PSFS values, e.g., during the high mobility time zone or when the location of the called MT is ambiguous i.e., for high mobility MU for which very recent interaction information does not exist. Fig. 3 depicts the PA selection for a call terminating to an ordinary mobility MU during a high mobility time zone. In this example, we assume that the " $M U$-related" paging information comprises the movement attraction point information and the most recent interaction information. As shown in the figure, the final PA comprises the adjacent BSP's to the one that the recent interaction information indicates (so as to increase the probability to page the called MU successfully). If the day time was also taken into account some of the adjacent BSP's could be excluded. For example, in the morning hours, the BSP's with numbers $4,11,19$, and 20 could be excluded, exploiting the fact that the called MU is on the way to work (given also 


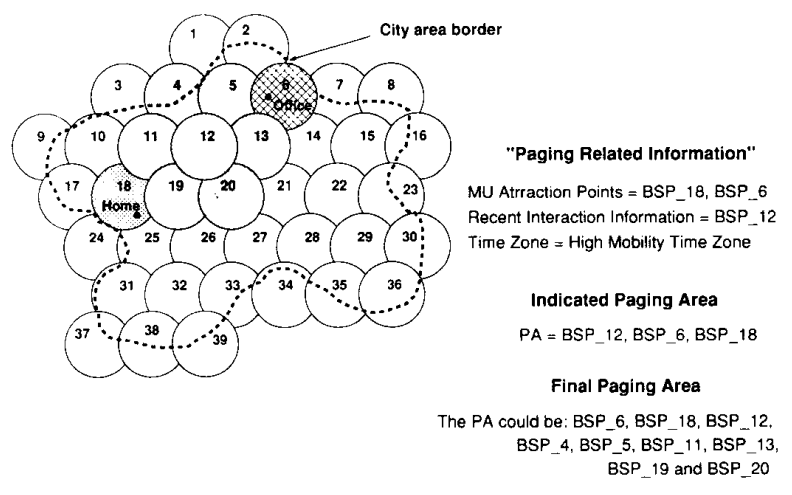

Fig. 3. Selection of the paging area based on "movement attraction points," "recent interaction information" and the "BSP topology" for an ordinary mobility MU during the high mobility hour.

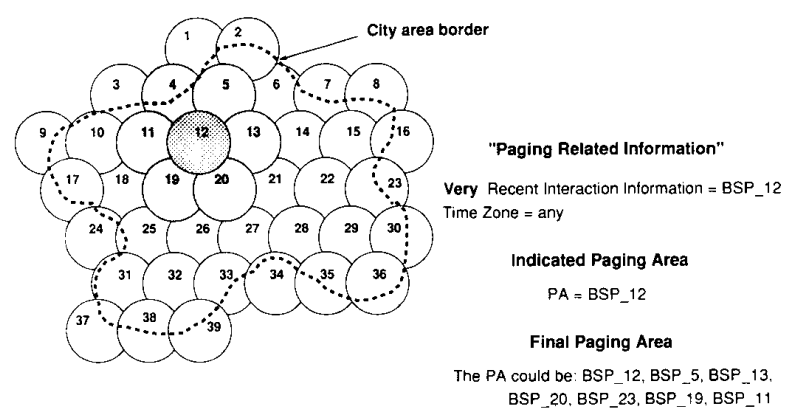

Fig. 4. Selection of the paging area based on a very "recent interaction information" and the "BSP topology" for a high mobility MU.

that it had a recent interaction at BSP no. 12). Fig. 4 depicts the PA selected for a call terminating to a high mobility MU for which a very recent interaction exists. Therefore, we select a PA that comprises the BSP indicated by the very recent interaction information plus all adjacent BSP's.

\section{III. "INTElligent" PAging Algorithm}

In this paragraph we present an example algorithm which describes the actions/operations that the network must take upon arrival of a new paging request. A generic view of the algorithm is depicted in Fig. 5. We may identify two phases:

\section{A. Selection Phase}

During this phase the network determines the "appropriate" paging strategy to be applied, i.e., either a single step paging strategy ("GSM-like" approach) or a multiple step one ("intelligent" paging) is selected. A detailed description of this phase is given in the flow chart of Fig. 6, while the basic steps of the algorithm are described in the sequel:

1) The network first examines whether paging is needed, by checking the current status of the terminal of the called MU (detached, attached, busy, free). If the terminal status is "detached," the paging procedure is canceled. On "busy," a relation between the terminal and the network

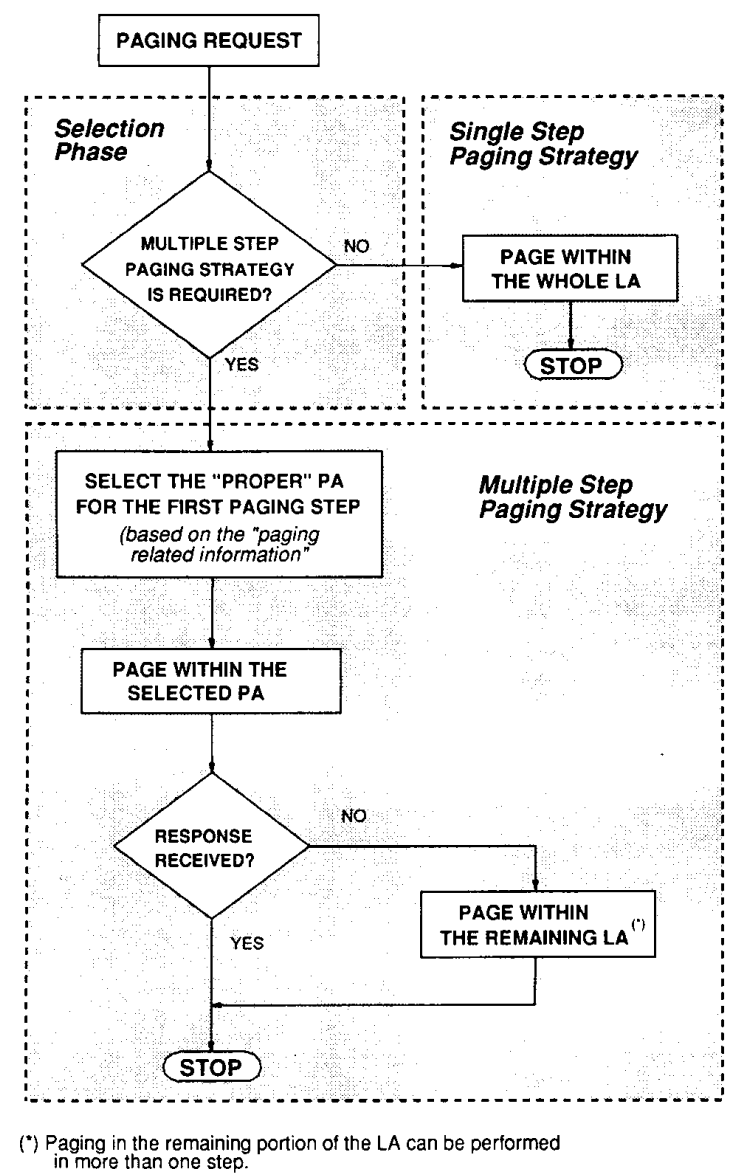

Fig. 5. The network operations upon arrival of a paging request.

already exists and therefore paging is not required. On "free," the network proceeds to paging.

2) The network examines whether the multiple step paging strategy should be applied or not. The decision is based on the current traffic conditions. If the current traffic load is lower that a certain threshold $\left(T_{0}\right)$, the single step paging strategy can be applied, otherwise, the multiple step is applied.

3) The network examines whether "MU-related" paging information is available and valid. If not available, the single step paging strategy is applied, otherwise, the multiple step paging strategy is applied.

\section{B. Execution Phase}

During this phase the actual execution of either the Single or the Multiple Step Paging Strategy is performed.

- Single Step Paging Strategy. If this is the case, paging is performed within the whole LA, i.e., all BSP's broadcast the paging message.

- Multiple Step Paging Strategy. The PA for the first paging step is determined. Then, paging is performed within the selected PA. If paging fails (due to unsuccessful prediction), another step is (or multiple steps are) required. The 


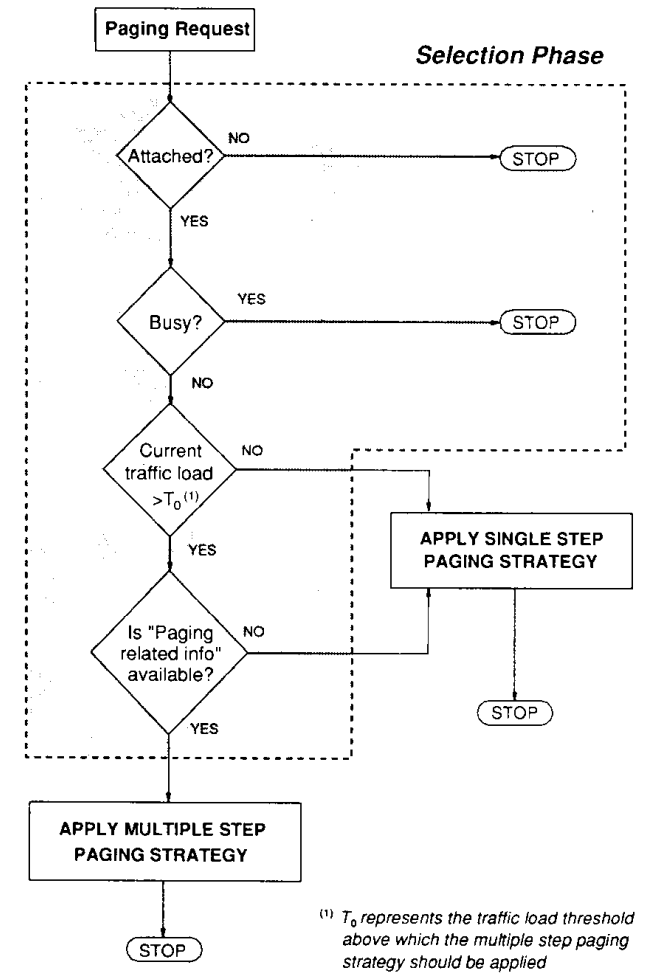

Fig. 6. Flow chart illustrating the way how the network decides whether the single or the multiple step paging strategy is required.

way how a PA can be formed according to Scenario 5 is depicted in the flow chart of Fig. 7 and described below:

- The group which the called MU belongs to is identified. For ordinary mobility MU's, information regarding the movement attraction points (BSPHome, BSP_WorkPlace, BSP_Other) is utilized. An initial PA that comprises the above mentioned BSP's is formed. That is, $\mathrm{PA}_{\text {init }}=B S P$ Home $+B S P_{-}$WorkPlace + BSP_Other. For high mobility MU's, the PA is equal to the whole LA, that is, $\mathrm{PA}_{\text {init }}=B S P_{-}^{*}$. Then,

- The information concerning the most recent interaction is taken into account. If the network deduces that such information is usable, the above determined PA is augmented to: $\mathrm{PA}=\mathrm{PA}_{\text {init }}+$ $B S P$ RecentInteraction for ordinary mobility MU's or changed to PA = BSPRecentInteraction for high mobility MU's. Then,

- The current time zone information (e.g., high, ordinary or low mobility time zone) is utilized to select the location (i.e., house, office, in motion) within which the called MU roams. This decision will either shrink the previous mentioned PA (low mobility time zone) or expand it (high mobility time zone) by taking into account the BSP topology. Finally,

- The first paging step is performed within the selected PA. If paging fails another set of BSP's is selected. The size of each PA is determined by the maximum allowable number of paging steps.

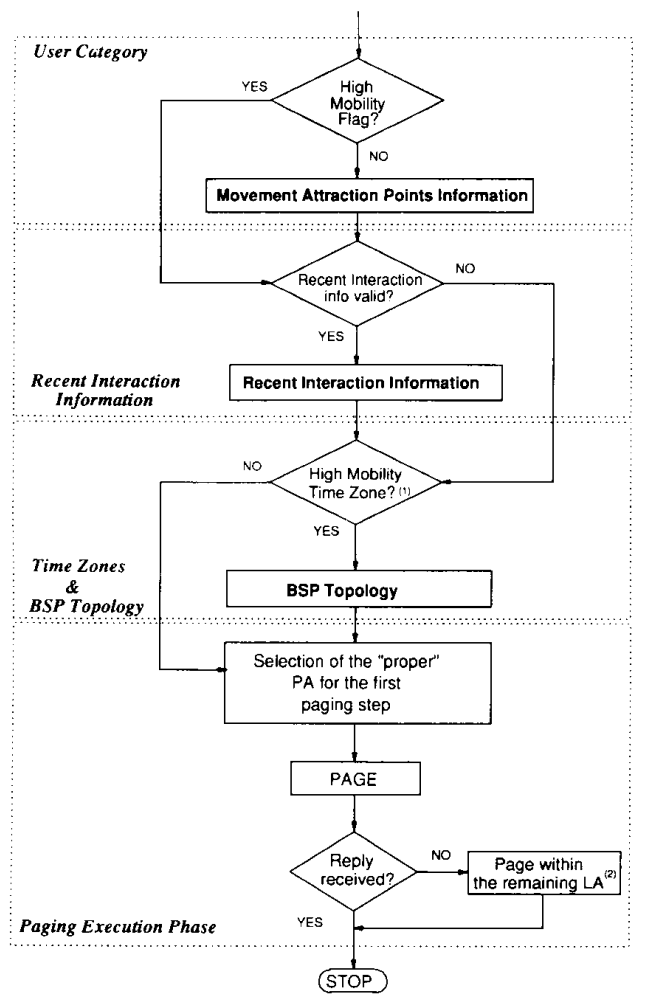

Fig. 7 . The sequence according to which the "paging related information" elements are utilized.

\section{Evaluation of the "Intelligent" Paging Strategy}

To assess the applicability of the above mentioned paging scenarios we used the city area simulation model first introduced in [6], which is also described in Appendix A. This model represents a typical city area consisting of four area types: the city center, the urban area, the suburban area and the rural area. In each area type the distribution of movement attraction points [houses, workplaces, other (e.g., shopping centers)] differs. In addition, three user categories have been assumed: a) high mobility users (e.g. taxis), b) working people, and c) housekeepers. For each user category a different mobility behavior is assumed. Finally, user movements are supported by a street-network consisting of four circular and four radial highways. For the network planning the following assumptions have been considered:

- the city area is covered by 32 BSP's i.e., 8 BSP's per area type,

- the city area is covered by a single LA.

The performance of the "intelligent" paging strategy is investigated through a series of specific simulated "cases" (see above described paging scenarios) shown in Table II. The simulated "cases" are compared with respect to: a) the quality of service they offer and b) the signaling load requirements (on the radio link) they impose. In particular, for each simulated case the following parameters are measured:

- the PSFS value versus user category and versus time, 
TABLE II

Definition of the Simulated Cases for the Performance Evaluation of the "Intelligent" Paging Strategy

\begin{tabular}{|c|c|}
\hline & Paging Strategy \\
\hline Scenario 1 & Paging is based on the recent interaction information ${ }^{12}$ \\
\hline Scenario 2 & $\begin{array}{l}\text { For ordinary \& low mobulity users scenario } 1 \text { is appled For high mobility users scenario } 1 \text { is } \\
\text { appled whenever the age of the recent interaction information is lower than a certain } \\
\text { "decision threshold" equal to } 10 \text { minutes. otherwise the "GSM-like" approach is applied }\end{array}$ \\
\hline Scenario 2a & $\begin{array}{l}\text { For both ordinary and low mobility users scenario } l \text { is applied For high mobility users the } \\
\text { GSM-like approach is always apphed }\end{array}$ \\
\hline Scenario 3 & $\begin{array}{l}\text { For high mobility users scenario } 2 \text { is applied for ordinary } \& \text { low mobility users the paging } \\
\text { area is selected based on (a) the recent interaction information and (b) the location of the } \\
\text { movement attraction points. }\end{array}$ \\
\hline Scenario 4 & $\begin{array}{l}\text { For high mobility users scenano } 2 \text { is applied. For ordinary \& low mobility users and during } \\
\text { low \& ordinary mobility time zones the paging area selection is based on the recent } \\
\text { interaction mformation and the location of the movement attraction points In this case } \\
\text { one of the movement attraction points is selected based on the current day time period. }\end{array}$ \\
\hline Scenario 5 & $\begin{array}{l}\text { During high mobility time zones and for all user categories the paging area selection is } \\
\text { based on (a) the recent interaction information' (If valid). (b) the location of the movement } \\
\text { attraction points and (c) the BSP topology The "decision threshold" regarding the recent } \\
\text { interaction information is assumed to be it minutes. Durng ordinary \& low mobility time } \\
\text { zones and for ordinary \& low mobllty users scenario } 4 \text { is applied }\end{array}$ \\
\hline Scenario $5 a$ & $\begin{array}{l}\text { Concerning scenario } 5 \text { the difference here is that the "decision threshold" is assumed to } \\
\text { be } 20 \text { minutes. }\end{array}$ \\
\hline Scenario $5 \beta$ & $\begin{array}{l}\text { Concerming scenario } 5 \text { the difference here is that the "decision threshold" is assumed to } \\
\text { be } 30 \text { munutes }\end{array}$ \\
\hline
\end{tabular}

- the maximum rate of paging messages transmitted per BSP (expressed as bitrate).

\section{A. Simulation Results}

Fig. 8 depicts the variation of the PSFS as well as the number of paging messages transmitted per BSP for the simulated "intelligent" paging cases described in Table II. The figures in boxes represent the percentage of the paging signaling load reduction with respect to the signaling load generated by the application of the "single step" paging strategy ("GSM-like" approach). From these percentages, it is obvious that the signaling reduction ranges approximately between $65 \%$ and $78 \%$. Notice that the PSFS increases with the detail of the "paging related information" while beyond a certain point the corresponding signaling load is rather stable. This is expected because as we increase the detail of the "paging related information" the number of BSP's selected also increases. Thus, the paging signaling reduction expected due to the PSFS increase is balanced by the increased number of BSP's selected for the first paging step. As shown in Fig. 8, the PSFS value varies between $72 \%$ ("case 1") and $98 \%$ ("case 5").

A specific study has been performed on the validity of the recent interaction information versus its age (see Scenario 1a in Section II-B-2). The variation of PSFS as well as the number of paging messages transmitted per BSP versus the decision threshold are depicted in Fig. 9. Obviously the PSFS decreases as the decision threshold increases. This is expected since the recent interaction information ceases being valid as the time passes. But notice that the paging signaling load also decreases as the decision threshold increases. This is strange at first glance, but it can be explained as follows: The recent interaction information is considered useless when its age exceeds the decision threshold. But when this happens,

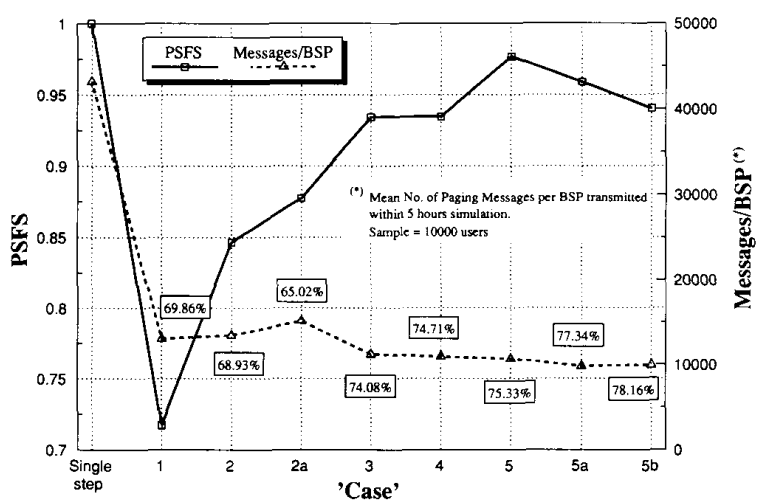

Fig. 8. Variation of PSFS and paging messages/BSP for various "cases."

the recent interaction information is not used, although there is a chance that it is valid despite its age. We illustrate this in Fig. 9 by showing in boxes the percentage of calls cases for which the "recent interaction information" age does not exceed the "decision threshold." Note that this percentage is only $30 \%$ when the age limit is 10 minutes, while it becomes $100 \%$ when there is no time limit.

Fig. 10 illustrates the variation of the PSFS as the simulation time runs. We may observe that:

- high PSFS values can be achieved during low mobility hours (e.g., busy hour)

- during rush hours the ambiguity of the user location results in low PSFS values for "cases 1 and 2." However, "cases 3, 4, and 5" result in satisfactory PSFS values even during rush hours.

Fig. 11 depicts the PSFS variation versus the MU category. PSFS values greater than $80 \%$ can be achieved for ordinary and low mobility users using low amount of paging related 


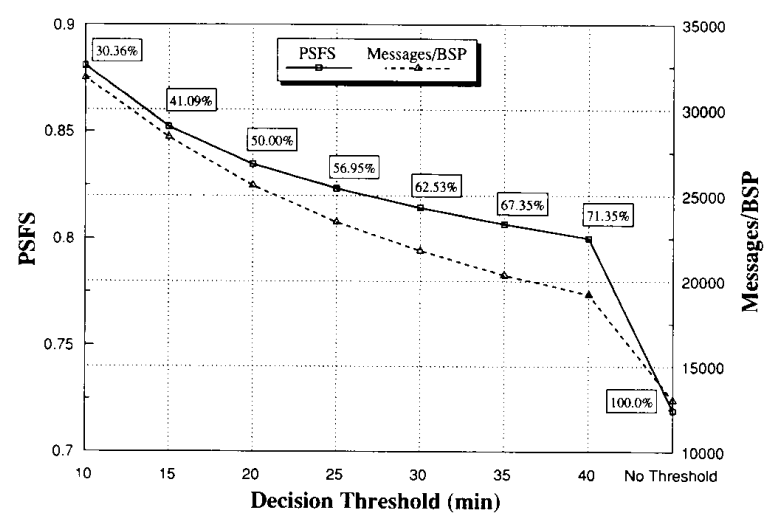

Fig. 9. PSFS variation and the paging messages/BSP versus decision threshold.

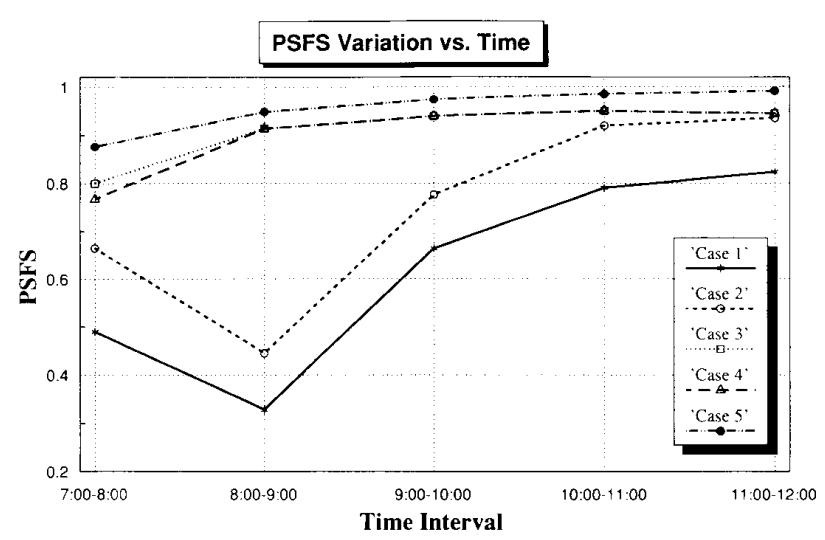

Fig. 10. PSFS variation for various "cases."

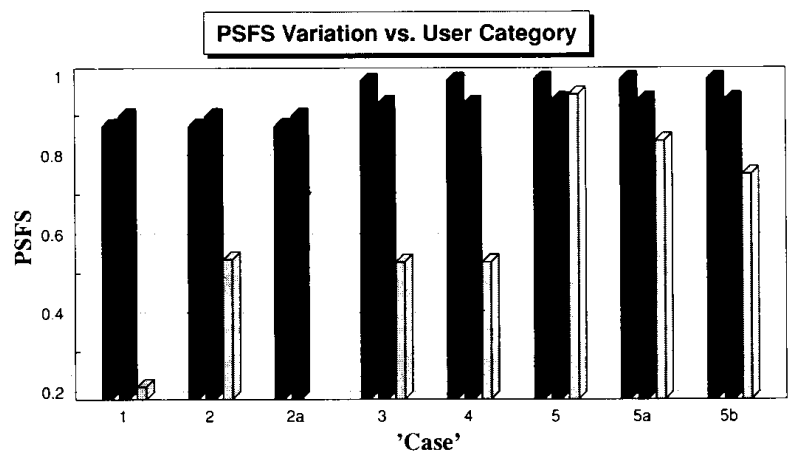

Fig. 11. PSFS variation versus the user category.

information (see "case 3"). On the contrary, for high mobility users satisfactory PSFS values can be achieved only in "cases $5,5 \mathrm{a}$, and 5b." The effectiveness of the latter scenarios depends on the decision threshold (see Table II). Fig. 12 depicts the PSFS variation for high mobility users versus the simulation time. It is clear that satisfactory PSFS values, during the whole simulation time period, can be achieved using "case 5."

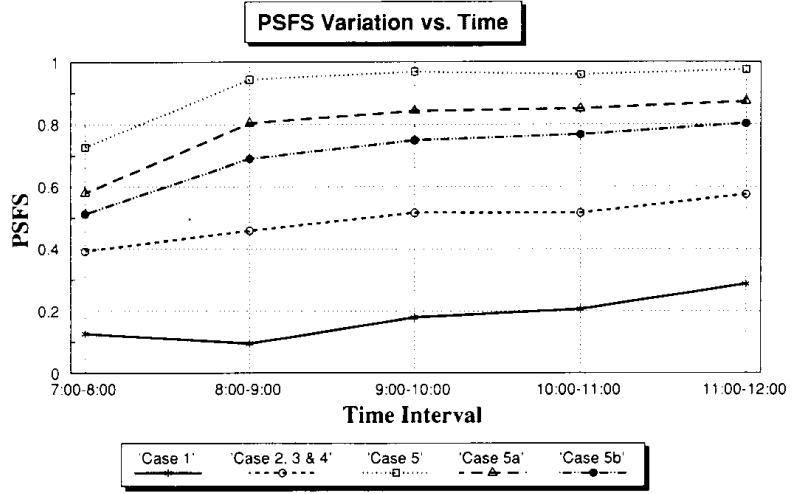

Fig. 12. PSFS variation for high mobility users versus time.

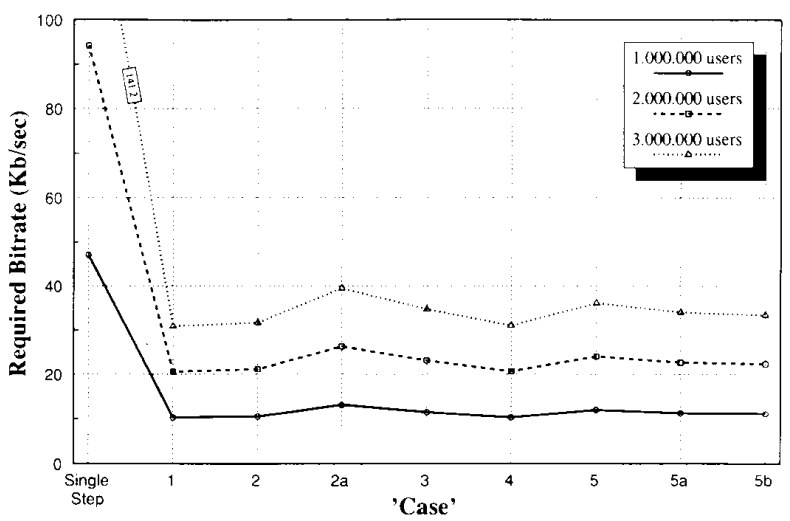

Fig. 13. Required bitrate for paging.

Finally, Fig. 13 illustrates the required bitrate for paging for the various "cases." Note that the results refer to the maximum paging signaling requirements. The paging message length is assumed equal to 100 bits. For the sake of comparison we depict the bandwidth requirements which correspond to the "GSM-like" approach in the case that the system serves 1, 2, and 3 millions of users. It is clear that the gains are more significant when the population size increases.

The performance of the "intelligent" paging strategies has been also investigated under abnormal mobility conditions. Those conditions are modeled by assuming that:

- all MU's are in movement

- all MU's behave as high mobility users.

In Figs. 14 and 15 the performance parameters (PSFS and required bitrate) for normal and abnormal mobility conditions are compared when the population of MU's is 2000000 . From these figures we may observe that:

- The PSFS value varies between $19.80 \%$ ("case 1") and $37.90 \%$ ("case 5").

- The application of the "intelligent" paging strategies under abnormal mobility conditions requires three times more bandwidth (Kbits/sec).

It is important to note that even under these (unrealistic) abnormal mobility conditions, the "intelligent" paging strategies still provide for paging signaling savings. 


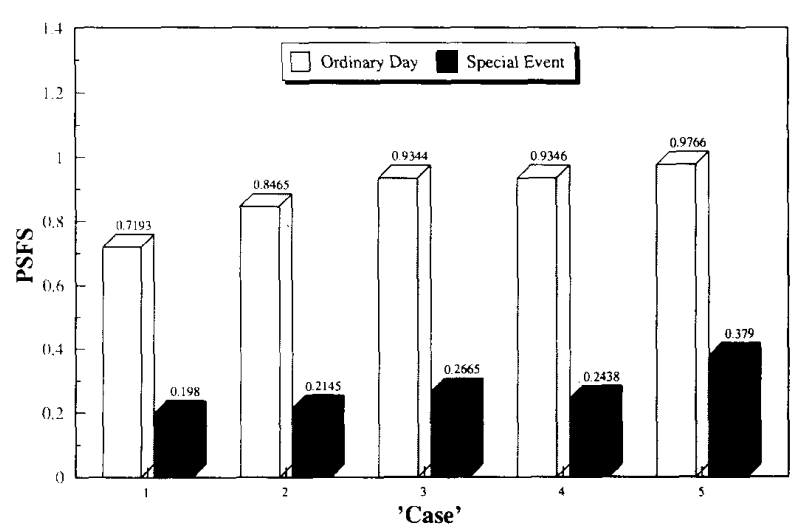

Fig. 14. The PSFS variation for normal and abnormal conditions.

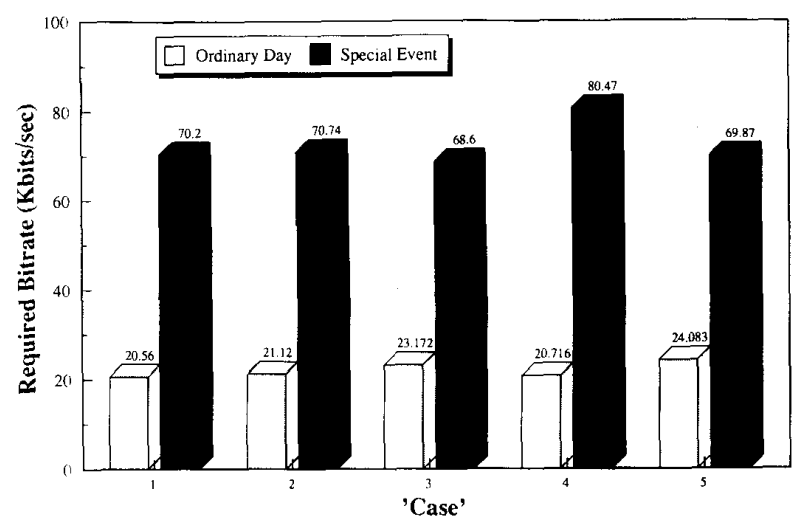

Fig. 15. Required bitrate variation for paging for normal and abnormal conditions.

\section{CONCLUSIONS}

In this paper, the performance of a representative set of "intelligent" paging strategy scenarios has been investigated for various day time periods (rush hours, busy hour, etc.) and user categories (high, ordinary and low mobility users) via a simulation tool which represents a realistic city area model. Simulation measurements focus on:

- the paging signaling load on the radio link (total messages, messages per BSP per hour).

- the PSFS value (overall, versus time, versus user category) which denotes the additional (paging) delay imposed to new calls due to unsuccessful paging attempts.

To assess the applicability of "intelligent" paging strategies, their performance has been also investigated under "abnormal" mobility conditions i.e., considering that all mobile users behave as high mobility users being thus in continuous movement.

Simulation results have shown that:

- "intelligent" paging achieves a paging signaling load reduction of the order of $70 \%$ or higher compared to the technique applied in GSM. Signaling savings can be achieved even under "abnormal' mobility conditions.

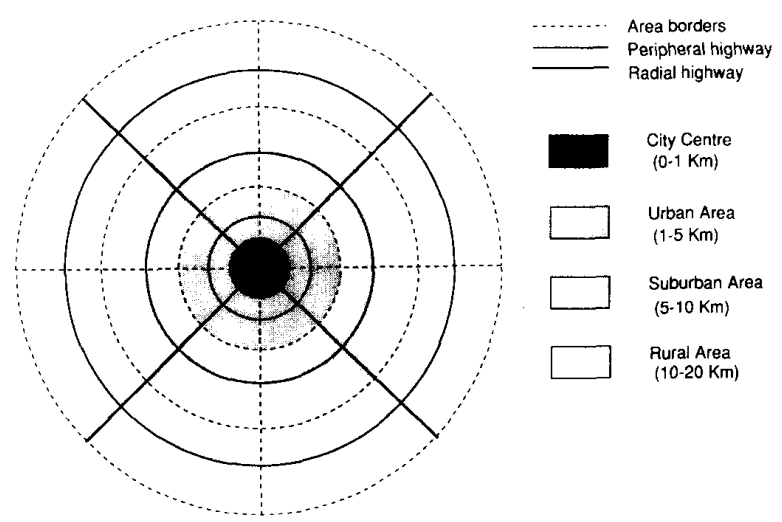

Fig. 16. The city area model consisting of area zones and highways.

- Significant increase of the number of mobile users does not affect the resulting signaling load considerably.

- Satisfactory predictions (e.g., PSFS $>90 \%$ ) at any day time period and for all user categories can be achieved when full "paging related information" is available ("case 5"). Less complex strategies (e.g., "case 2")—which implies lower PSFS values-does not affect neither the paging signaling load nor the call set-up delay considerably.

To conclude, we have shown that "intelligent" paging achieves a significant reduction of the paging signaling load compared to the technique applied in GSM. This allows for the definition of larger LA's and therefore may lead to the minimization of the location updating signaling load (e.g., a single LA may cover a whole city area). The penalty paid is: a) additional storage space is required in the network database (the " $M U$-related" paging information) and b) some additional functionality is needed to process the "paging related information." However, neither the storage nor the processing requirements can be considered significant as far as the applicability of the "intelligent" paging strategies is concerned.

\section{APPENDIX}

\section{A. Geographical Area Model}

A city area is the basis for the whole study. The city area is assumed to have a radius of $20 \mathrm{~km}$ and is modeled as a set of area zones and a set of highways supporting movements within the city area (see Fig. 16). In our city model we consider four area types: the city center, the urban area, the suburban area and the rural area. Our city model consists of 32 area zones ( 8 per city area type), four circular highways (one per area type) and four radial highways. Note that each area zone is covered by one BSP. The city area population is assumed to be 6 million people and the MU penetration rate is assumed to be $50 \%$ (i.e., there are 3 million MU's within the city area). In our simulation tool we simulate a sample of $10000 \mathrm{MU}$ 's roaming within this city area. 


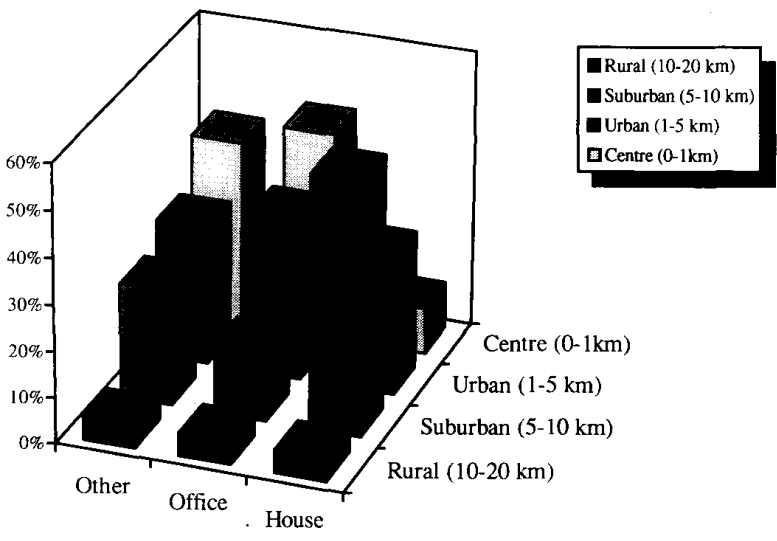

Fig. 17. The distribution of the movement attraction points over the city area.

\section{B. Movement Attraction Points}

In each area zone the following types of movement attraction points are assumed to exist:

- Houses

- Workplaces

- Other (shopping centers, parks, etc.).

The distribution of the movement attraction points over the city area zones is uniform within the same area type. In Fig. 17. the distribution used in our simulation model is presented.

\section{MU Categories Based On Their Mobility Behavior}

Mobile users, according to the mobility behavior they exhibit, are grouped into three categories for simplicity denoted as: a) working people, b) housekeepers, and c) high mobility users. Additional MU categories can be envisaged by taking into account the transportation means they use (private car, public transportation) as shown in Fig. 18. In addition the percentages of the various transportation means utilized by each MU category are depicted in Fig. 18.

\section{Time Zones}

The simulation time is 5 hours: from 7:00 a.m. to 12:00 p.m. This time period is selected so as to include both the rush hours and busy hour (see Fig. 19). Three time zones are considered: a) 7:00-9:00 a.m. (the rush hours), 9:00-11:00 a.m., 11:00 a.m.-12:00 p.m. (the busy hour).

\section{E. Initial MU Distribution Over The City Area}

MU's are initially distributed over the city area by assuming that most of them are situated at their residence. The distribution of residence places used in our simulation model is shown in Fig. 17.

\section{F. MU Movements}

The way that MU's are assumed to perform movements depends on the MU degree of mobility, the distribution of the movement attraction points as well as the highway topology. For every MU located in a movement attraction point

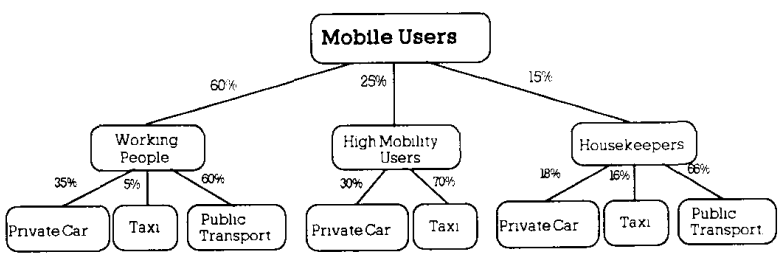

Fig. 18. Categorization of mobile users according to their mobility behavior.

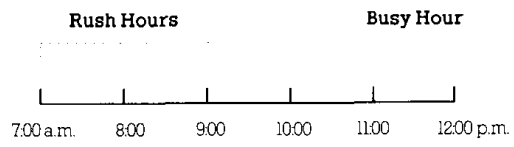

Fig. 19. The simulation time period.

we determine the residence time. When the residence time has expired a movement destination is selected. Given the movement destination, the route via which the MU will move is determined. In general a movement from an area zone to another can be discriminated into two parts (due to the highway topology):

- radial movement,

- circular movement.

In our model, movements from area zone to area zone follow the shortest path. If an MU moves toward an inner city area zone, then first moves radially and then circularly. If the MU moves toward an outer city ring, then first moves circularly and then radially.

1) Movement Algorithm for Working People: Working people initiate a movement within a time interval uniformly distributed between 7:00 a.m. and 7:30 a.m. After a short period of walking-depends on the transportation means they use for the movement (5-15 min.)-the MU rides on the vehicle that is about to carry him/her to his/her workplace (destination). The vehicle may be either the private car, a taxi or some public transportation mean. In the latter case, the MU may use more than one transportation means. After another short period of walking, he/she reaches his/her workplace.

2) Movement Algorithm for Housekeepers: We assume that every housekeeper initiates a movement in a time interval uniformly distributed between 7:30 a.m. and 9:30 a.m. We discriminate two kinds of movements: a) those which take place within the area zone that the MU residence locates and b) those which are destined to a different area zone. For movements of type a), MU's are regarded as pedestrians for a time interval between 0.5 and 2 hours. For movements of type b), MU's use either their private car, a taxi or some public transportation means in order to move to another area zone. The "residence time" within the destination area zone is distributed between 0.5 and 3 hours (are then regarded as pedestrians moving to banks, shops or other attraction points in this area zone).

3) Movement Algorithm for High Mobility Users: It is assumed that MU's belonging to this category are already in motion, having a destination when the simulation starts. As soon as a movement completes, a new destination is assigned to them. This procedure is repeated during the whole 


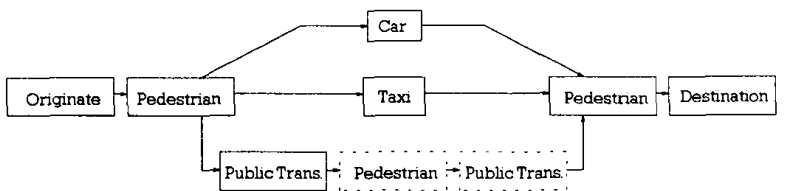

Fig. 20. Movement algorithm for working people and housekeepers.

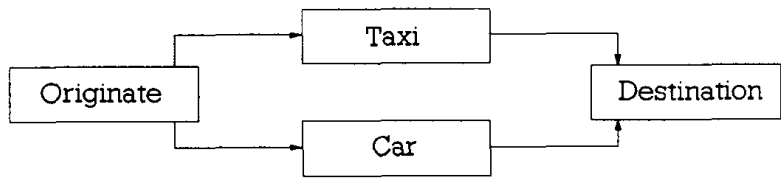

Fig. 21. Movement algorithm for high mobility Users.

TABLE III.

The Range of Vehicle Veloctties in Conjunction with the Area Type

\begin{tabular}{|c|c|c|}
\cline { 2 - 3 } \multicolumn{1}{c|}{} & \multicolumn{2}{c|}{ KIND OF VEHICLE } \\
\hline AREA TYPE & $\begin{array}{c}\text { PUBLIC TRANSPORTATION } \\
\text { MEDIA }\end{array}$ & CAR or TAXI \\
\hline City Centre & $5-15 \mathrm{~km} / \mathrm{h}$ & $10-20 \mathrm{~km} / \mathrm{h}$ \\
\hline Urban Area & $10-30 \mathrm{~km} / \mathrm{h}$ & $15-40 \mathrm{~km} / \mathrm{h}$ \\
\hline Suburban Area & $30-70 \mathrm{~km} / \mathrm{h}$ & $40-80 \mathrm{~km} / \mathrm{h}$ \\
\hline Rural Area & $60-80 \mathrm{~km} / \mathrm{h}$ & $60-100 \mathrm{~km} / \mathrm{h}$ \\
\hline
\end{tabular}

simulation process. Figs. 20 and 21 depict the above described movement algorithms.

\section{G. Mobility Conditions}

The average pedestrian speed is assumed to be $5 \mathrm{~km} / \mathrm{h}$ everywhere. The vehicle (private car, taxi, bus) velocity is selected uniformly, based on the ranges which in conjunction with the area types are given in Table III.

\section{H. Traffic Conditions}

In our simulation we consider the arrival of both incoming and outgoing calls to be Poisson. Table IV gives the call arrival rate (both incoming and outgoing) for each MU category during three periods: a) 7:00 a.m.-9:00 a.m., b) 9:00 a.m.-11:00 a.m., and c) 11:00 a.m.-12:00 p.m. In addition, we show the percentage of incoming and outgoing calls for each MU category. Note that, in the case of high mobility users the rate of incoming calls is higher than the outgoing rate. This is because we assume that high mobility users are involved in jobs (e.g., taxis) where incoming calls are for both private and business communication while outgoing calls are mainly for private communication.

\section{REFERENCES}

[1] CCITT SG I, Draft Recommendation F 851, Universal Personal Telecommunications, Service Description, Version 6, Kitakyushi, Nov. 1991.

[2] RACE 2066 Mobile Networks (MoNet) project, CEC Deliverable No R2066/BT/PM2/PS/070/b2, UMTS System Structure Document, Issue 2.0, Dec. 1994

[3] M. Mouly and M.-B. Pautet, "The GSM system for mobile communications," published by the authors, 1992.
TABLE IV

Call arrival Rate (CALls/MU/H) For all

MU Categories Versus Day Time Period

\begin{tabular}{|c|c|c|c|c|}
\hline \multirow[b]{2}{*}{ MU Category } & \multicolumn{3}{|c|}{ CALL ARRIVAL RATE (calls/MU/h) } & \multirow[b]{2}{*}{ Incoming-Outgoing } \\
\hline & 7:00-9:00 & $9.00-1100$ & $1100-12.00$ & \\
\hline Working People & 0.5 & 20 & 3.5 & $50 \%-50 \%$ \\
\hline Housekeepers & 0.5 & 15 & 20 & $50 \%-50 \%$ \\
\hline High Mobility Users & 10 & 20 & 3.5 & $70 \%-30 \%$ \\
\hline
\end{tabular}

[4] B. Samadi and W. S. Wong, "Optimization techniques for location area partitioning," in 8th ITC Specialist Seminar on Universal Personal Telecommun., Geneva, Oct. 1992.

[5] S. Okasaka, S. Onoc. Syuji Yasoda, and A. Maebara, "A new location updating method for digital cellular systems," in 41st VTS Conference, St. Louis, Missouri, May 1991.

[6] J. G. Markoulidakis, G. L. Lyberopoulos, D. F. Tsirkas, and E. D. Sykas, "Evaluation of location area planning scenarios in future mobile telecommunication systems," Wireless Networks, vol. 1, no. 1, pp. 17-29, Jan. 1995 .

[7] A. Bar-Noy and I. Kessler, "Tracking mobile users in wireless communications networks," in Proc. INFOCOM '93, 1993, pp. 1232-1239.

[8] B. Awerbuch and D. Peleg, "Concurrent on-line tracking of mobile users," in Proc. ACM SIGCOM Symposium on Communication, Architecture and Protocols, Oct. 1991, pp. 221-233.

[9] V. Anantharam, M. L. Honig, U. Madhow, and V. K. Wei, "Optimization of a signaling hierarchy for mobility tracking in personal communications networks," in Inter. Teletraffic Congress, India, Nov. 1993, pp. 45-51.

[10] S. Tabbane, "Evaluation of an alternative location strategy for future high density wireless communications systems," WINLAB Tech. Report, July 1992.

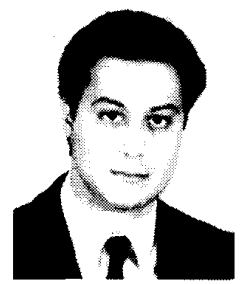

George L. Lyberopoulos (S'90-M'93) was born in Athens, Greece, on April 26, 1965. He received the undergraduate Diploma degree in electrical engineering from the University of Thrace in 1989, and the Ph.D. degree in electrical engineering from the National Technical University of Athens (NTUA) in 1994.

Since 1989 he has been involved in many projects for the European Communities: (R1044/2.10 Broadband User/Network Interface, Multi-Project Task Group on Numbering and Addressing, R1043 Mobile Project, R2066 Mobile Networks project, etc.). His research interests are in the areas of resource allocation and performance evaluation of integrated networks, statistical multiplexing and switching techniques, local area networks and digital mobile communication systems.

Dr. Lyberopoulos is a member of the Technical Chamber of Greece.

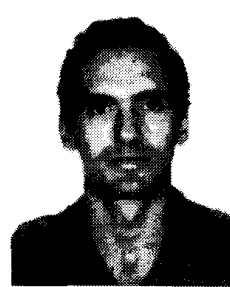

John G. Markoulidakis was born in Athens, Greece, in 1966. He received the undergraduate Diploma degree in electrical engineering from the National Technical University of Athens (NTUA) in 1990 , and the Ph.D. degree in electrical engineering from the NTUA in 1994.

Since 1990 he has been involved in several projects for the European Communities (RACE-1043 Mobile Project, RACE-2066 Mobile Networks, etc.). His research interests are in the areas of resource allocation and performance evaluation of integrated digital networks and mobile telecommunication systems.

Dr. Markoulidakis is a member of the Technical Chamber of Greece. 


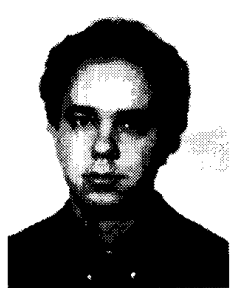

Dimitrios V. Polymeros was born in Athens, Greece, on April 22, 1969. He received the undergraduate Diploma degree in electrical engineering from the National Technical University of Athens (NTUA) in 1993. He is currently studying toward the Ph.D. degree in the Department of Electrical Engineering, in the NTUA, in the area of Telecommunications.

Since 1993 he has been involved in the RACE2066 Mobile Networks research project for the European Communities. His research interests are in the areas of mobile telecommunication systems (mobility management and protocols construction).

Mr. Polymeros is a member of the Technical Chamber of Greece.

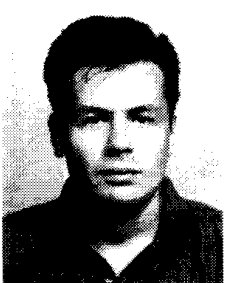

Dimitrios F. Tsirkas was born in Athens, Greece, on July 16,1970 . He received the undergraduate Diploma degree in electrical engineering from the National Technical University of Athens (NTUA) in 1993. He is currently studying toward the Ph.D. degree in the Department of Electrical Engineering, in the NTUA, in the area of Telecommunications. Since 1993 he has been involved in the RACE2066 Mobile Networks research project for the European Communities. His research interests are in the areas of performance evaluation of integrated digital networks and mobile telecommunication systems. Mr. Tsirkas is a member of the Technical Chamber of Greece.

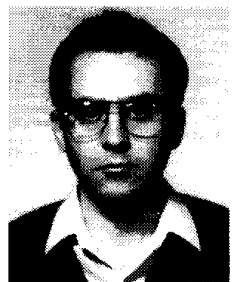

Efstathios D. Sykas (S'79-M'83) was borm in Athens, Greece, in 1956. He received the Dipl.-Ing. and Dr.-Ing. degrees in electrical engineering both from the National Technical University of Athens, Greece, in 1979 and 1984, respectively.

From 1979-1984 he was a Teaching Assistant, during 1986-1988 he was a Lecturer, then an Assistant Professor, 1988-1992, and now an Associate Professor in the Computer Science Division, Department of Electrical Engineering, National Technical University of Athens (NTUA). During 1985-1986 he worked at the Research Center of Crete, Greece, for the development of a command and control information system. Duties inside NTUA include teaching of graduate courses on computer networks, telephony and communication systems and a post-graduate course on signaling protocols in broadband networks.

His research interests are in the fields of broadband communications, integrated services networks, mobile and personal communications, intelligent networks, signaling protocols, numbering and addressing, directory systems, computer communication networks, multiple access protocols, LAN's, MAN's, WAN's, switching techniques (packet, circuit and hybrid switching systems) analysis and synthesis of communication networks, routing, flow control and load sharing, queuing theory, performance evaluation of data networks and computer systems. He has over fifty publications in the above areas. He received several national and international academic awards.

He has participated in several RACE projects: RDP 2023 - ATD technique, RDP 1006 - Subscriber premises networks, R1043 Mobile, R1022 Technology for ATD, R1014 ATMOSPHERIC, R2016 STRATOSPHERIC, and R1044 (Customer Service Functions) CSF, R2066 MONET, R2023 UNOM, R2081 TRIBUNE. He has participated in six national research programs dealing with data communications networks. These included development of modeling, simulation, analysis, design and performance measurement packages for packet and circuit switched data networks.

Dr. Sykas is a reviewer for several international journals and a member of IEEE Standard 802 Committee. He is a member of ACM and the Technical Chamber of Greece. 